\title{
Unifying variational methods for simulating quantum many-body systems
}

\author{
C.M. Dawson, ${ }^{1,2}$ J. Eisert, ${ }^{1,2}$ and T.J. Osborne ${ }^{3}$ \\ ${ }^{1}$ Blackett Laboratory, Imperial College London, Prince Consort Road, London SW7 2BW, UK \\ ${ }^{2}$ Institute for Mathematical Sciences, Imperial College London, Prince's Gate, London SW7 2PE, UK \\ ${ }^{3}$ Department of Mathematics, Royal Holloway University of London, Egham, Surrey TW20 OEX, UK
}

(Dated: October 25, 2018)

\begin{abstract}
We introduce a unified formulation of variational methods for simulating ground state properties of quantum many-body systems. The key feature is a novel variational method over quantum circuits via infinitesimal unitary transformations, inspired by flow equation methods. Variational classes are represented as efficiently contractible unitary networks, including the matrix-product states of density matrix renormalization, multiscale entanglement renormalization (MERA) states, weighted graph states, and quantum cellular automata. In particular, this provides a tool for varying over classes of states, such as MERA, for which so far no efficient way of variation has been known. The scheme is flexible when it comes to hybridizing methods or formulating new ones. We demonstrate the functioning by numerical implementations of MERA, matrix-product states, and a new variational set on benchmarks.
\end{abstract}

PACS numbers: 03.65.Bz, 89.70.+c

Quantum many-body systems pose some of the most difficult challenges in modern physics, and many examples remain inaccessible to analysis. Of the many methods that have been devised as attempts to meet these challenges, one of the most successful has been the density matrix renormalization group (DMRG) [1]. The DMRG was originally conceived as a numerical technique for iteratively constructing the ground or low-energy states of a Hamiltonian, so that the system's Hilbert space is truncated and the difficulties associated with exponentially increasing dimension are avoided. A more recent interpretation of the DMRG has cast it as a variational method over matrix product states [2, 3, 4, 5, 6], and this shift in emphasis has stimulated much work on extending its applicability.

Matrix product states are expected to provide good approximations to the ground states of one-dimensional noncritical systems [7], however in other cases it is expected that alternative variational sets will be required. Motivated by this, new classes have been introduced, such as projected entangled pair states [4] and weighted graph states [9] for higher-dimensional lattices, while multi-scale entanglement renormalization (MERA) [10] and contractor renormalization [11] may be more appropriate for critical systems. At first sight it may appear that these numerical approaches to quantum many-body systems have little in common with each other. Moreover, the specification of a variational class is only a first step - we also require an effective method of finding the best description of the system's ground or low-energy states within that class.

In this letter, we provide a unifying picture for several of these variational methods: We show that by (i) recasting state classes as quantum circuit classes (unitary networks) one can formulate a (ii) general purpose variational method, related to the framework of flow equations [12]. We shall see that to provide a working numerical method, it is sufficient that the propagation of each Hamiltonian term can be efficiently tracked on a classical computer. The con-

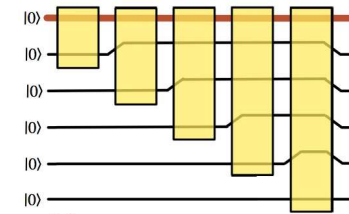

(a)

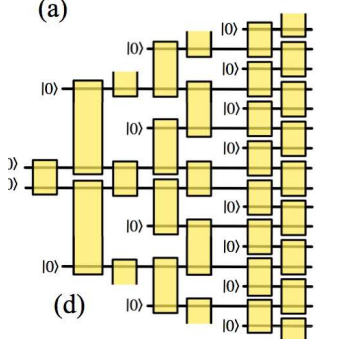

(b)

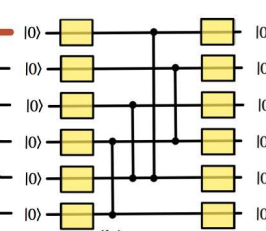

(b)

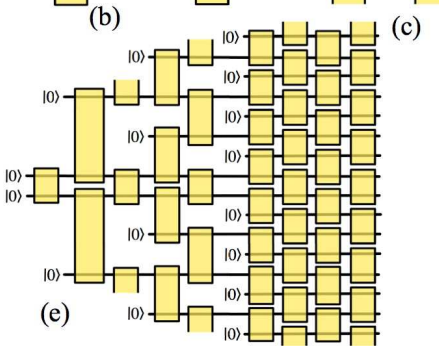

FIG. 1: (a) An example of what is here called a staircase circuit. The upper system here is $d$-dimensional. Further examples of unitary networks considered here include: (b) Unitary network of a weighted graph state up to local deformations. (c) Quantum cellular automata with Margolus partitioning. (d) Quantum circuit of MERA including disentangling operations, (e) A new variant combining (c) and (d) ("extended MERA").

tractibility properties of the state classes mentioned above translate immediately into an analogous property on their corresponding circuits. Our approach - a flow equation approach to variational simulations - may be regarded as an optimal control approach [12, 13] to varying efficiently contractible networks describing variational states of quantum many-body systems. It is flexible enough to hybridize known methods or to construct new ones, and provides a first efficient way of variation over MERA.

Variational sets. - We begin with the casting of variational sets as unitary networks, which provides the basis for the flow equations approach. Given $N$ spins (and possibly ancillary systems) consider a family of states $S_{d}=$ 
$\left\{U|\mathbf{0}\rangle: U \in \mathcal{U}_{d}\right\}$, where $U \in \mathcal{U}_{d}$ is a set of unitary networks characterized by some refinement parameter $d$, and $|\mathbf{0}\rangle$ denotes the state vector with all spins down. The refinement parameter plays the role, e.g., of the auxiliary dimension in matrix-product states (MPS). These networks consisting of gates $U=\prod_{j=1}^{M} U_{j}$ have to satisfy the condition that correlators of the form $\left\langle\mathbf{0}\left|U^{\dagger} O_{1} \ldots O_{k} U\right| \mathbf{0}\right\rangle$ can be efficiently computed for any $k$, that is with effort polynomial in $N$ and $d$. Before turning to the variational method we will discuss a number of important examples of states that can be discussed in this framework. We begin by considering the matrix-product states of DMRG. For a system consisting of $N$ spins, we introduce a $d$-dimensional ancilla system, and consider a circuit consisting of $N$ gates $U_{j}$ which act on both the ancilla system and qubit $j$, giving a "staircase" form, see Fig.11a). By projecting the output of such circuits onto some basis state of the ancillary system or by disentangling it, we obtain any matrix-product state on the spins. A second important example is the MERA class, see Fig. 1(d). This circuit is arranged in a tree structure with $\log N$ distinct layers, each of which introduces new spins into the circuit via two sets of gates known as isometries and disentanglers [10]. An analogous circuit is also possible using 2D binary trees. Further examples of quantum circuit classes are weighted graph states, where the refinement parameter $d$ is defined by the non-zero entries of the adjacency matrix of the weighted graph, and quantum cellular automata [14], the finite depth $d$ being the refinement parameter, and new variants as depicted in Fig. 1.

Flow equations as a unifying method of variation. Before we introduce the method of variation, let us first remind ourselves of flow equation ideas. Consider a continuous transformation of an initial Hamiltonian $H$ $H(t)=U(t)^{\dagger} H U(t)$, where $U(t)$ is defined via a Hermitian generator as the time-ordered integral $U(t)=$ $\mathcal{T} \exp \left(-i \int_{0}^{t} G(s) d s\right)$. The derivative of $H(t)$ is given by $\partial_{t} H=-i[G(t), H(t)]$. A familiar example from optimal control theory chooses $G(t)=i[K, H(t)]$, where $K$ is a real diagonal matrix with unique entries. In this case $H(t)$ will converge to a diagonal matrix of eigenvalues as $t \rightarrow \infty$, with the columns $U(t)$ the corresponding eigenvectors. This is often referred to as double-bracket flow [12]. A straightforward application to quantum many-body systems is impractical, as the flow will in general transform the Hamiltonian into one having exponentially many terms. The key to these methods is to truncate the resulting systems of differential equations in a perturbative fashion that is a good approximation for small perturbations.

However, we are not aiming for approximate analytical expressions here. Consider a quantum circuit as be a sequence of $M$ gates $U_{j}(t)$, each of which is continuously parameterized with infinitesimal generator $G_{j}(t)$ beginning with some arbitrary $U_{j}(0)$. Write the overall unitary implemented by the circuit as $U(t)=\prod_{j=1}^{M} U_{j}(t)$, and consider the expectation of some many-body Hamiltonian $E(t)=\left\langle\mathbf{0}\left|U(t)^{\dagger} H U(t)\right| \mathbf{0}\right\rangle$. A circuit class is defined here by a specification of the locations of each gate $U_{j}(t)$, and the best approximation to a ground state within a given class is the circuit that minimizes the expectation $E(t)$. Within the framework of flow equations, we will show how one can choose optimal generators $G_{j}(t)$ for each gate. Differentiating the expectation we get $\partial_{t} E=2 \mathfrak{R e}\left\langle\mathbf{0}\left|U^{\dagger} H \partial_{t} U\right| \mathbf{0}\right\rangle$, and our first goal is to minimize this derivative subject to the Hilbert-Schmidt constraints $\operatorname{tr}\left[G_{j}(t)^{\dagger} G_{j}(t)\right]=\varepsilon$, i.e., the generators remain “infinitesimal". For $U(t)=\prod_{j=1}^{M} U_{j}(t)$, we find

$$
\partial_{t} U=-i \sum_{j=1}^{M}\left(\prod_{k=j+1}^{M} U_{k}\right) G_{j}\left(\prod_{k=1}^{j} U_{k}\right) .
$$

Note the convention whereby $\prod_{j=1}^{M} U_{j}$ is ordered as $U_{M} \cdots U_{2} U_{1}$, and the other way round for $\prod_{j=M}^{1} U_{j}$. We can substitute this back and minimize on a term-by-term basis at each point $t$ of the flow. Let $\left\{B^{b}\right\}$ be an appropriate orthonormal Hermitian operator basis, and expand the $j$-th generator as $G_{j}(t)=\sum_{b} g_{j, b} B^{b}$, with $g_{j, b}$ real. Now define, for the given Hamiltonian $H$,

$$
\Gamma_{j, b}(t)=\left\langle\mathbf{0}\left|U^{\dagger} H\left(\prod_{k=j+1}^{M} U_{k}\right) B^{b}\left(\prod_{k=1}^{j} U_{k}\right)\right| \mathbf{0}\right\rangle .
$$

Each term of the derivative with this parametrization is $\left[\partial_{t} E\right]_{j}=2 \sum_{b} g_{j, b} \mathfrak{R e}\left[\Gamma_{j, b}(t)\right]$, and the constraints of the minimization problem are $\sum_{b} g_{j, b}^{2}=\varepsilon$. The Lagrange multiplier condition for a minimum is then simply $g_{j, b}=$ $-2 \mathfrak{R e}\left[\Gamma_{j, b}(t)\right] / \lambda$. The following method of evaluating the optimal generator avoids calculating the quantities $\Gamma_{j, b}(t)$ for each basis element $B^{b}$ : Writing $G_{j}$ out in its operator basis we have $G_{j}(t)=-(2 / \lambda) \sum_{b} \mathfrak{R e}\left[\Gamma_{j, b}(t)\right] B^{b}$. Recall that the real part appears because we are taking an expectation of an operator and its Hermitian conjugate. It will be convenient to set $G_{j}=-(2 / \lambda)\left(F_{j}+F_{j}^{\dagger}\right)$, with

$$
F_{j}=\operatorname{tr}\left[\left(\prod_{k=1}^{j} U_{k}\right)|\mathbf{0}\rangle\langle\mathbf{0}| U^{\dagger} H\left(\prod_{k=j+1}^{M} U_{k}\right) B_{j}^{b}\right] B_{j}^{b},
$$

which can after some steps be written in terms of a partial trace over the subsystems $R_{j}$ not acted on by the gate $U_{j}$

$$
F_{j}=\operatorname{tr}_{R_{j}}\left[\left(\prod_{k=1}^{j} U_{k}\right)|\mathbf{0}\rangle\langle\mathbf{0}| U^{\dagger} H\left(\prod_{k=j+1}^{M} U_{k}\right)\right] .
$$

The utility of this expression depends on whether or not we are able to efficiently calculate the partial trace, which depends on the structure of the circuit class $\prod_{j=1}^{M} U_{j}$ and on the Hamiltonian $H$. Accordingly, we now introduce contraction techniques able to cope with such expressions [15]. 
(a)

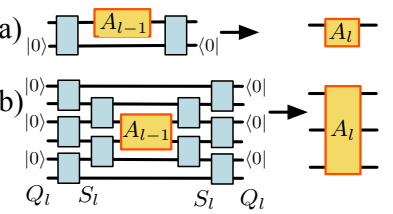

(c)

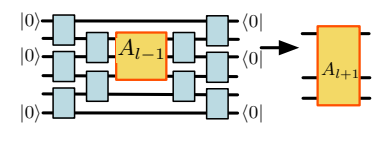

(d)

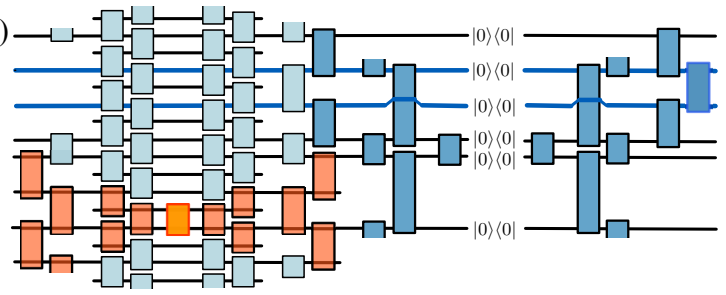

FIG. 2: Contraction rules that are used in the procedure of finding the optimal generators (a-c), and a circuit correponding to Eq. (3) for MERA (d). The red and blue shading indicate the two causal cones that are encountered when evaluating the partial trace.

Contraction. - We begin by reviewing some of the basic ideas of contraction and introduce a language for describing them. What we here call a standard contraction problem calls for the evaluation of an expectation of the form $\left\langle 0\left|U^{\dagger} A U\right| 0\right\rangle, U$ being a quantum circuit $\prod_{j=1}^{M} U_{j}$ acting on $N$ spins (and any ancillae), and $A$ some observable. A contraction procedure is a sequence of maps that construct operators $A_{0}, A_{1}, \ldots, A_{L}$ with $A_{0}=A$ and

$A_{l}=\left(\left\langle\left.\mathbf{0}\right|_{Q_{l}}\right) \otimes \mathbb{1}\right)\left(\prod_{j \in S_{l}} U_{j}\right)^{\dagger} A_{l-1}\left(\prod_{j \in S_{l}} U_{j}\right)\left(|\mathbf{0}\rangle_{Q_{l}} \otimes \mathbb{1}\right)$.

Here $Q_{l}$ and $S_{l}$ denote subsystems and subcircuits respectively, and are chosen so that at the final step we obtain $\left\langle\mathbf{0}\left|A_{L}\right| \mathbf{0}\right\rangle=\left\langle\mathbf{0}\left|U^{\dagger} A U\right| \mathbf{0}\right\rangle$. The contraction is said to be efficient if the dimensions of the operators $\prod_{k \in S_{j}} U_{k}$ scale at most polynomially in the number of spins $N$.

The key point here is that we may evaluate such an expectation (or similarly a trace or partial trace) without ever having to deal with the overall unitary $U$, whose dimension is in general exponential in $N$. As an example, suppose we have a two-body Hamiltonian term $H_{j, j+1}$, and wish to evaluate $\left\langle 0\left|U^{\dagger} H_{k, k+1} U\right| 0\right\rangle$ for $U$ a staircase circuit. Then we set $A_{0}=H_{k, k+1}$ and iterate $A_{l}=$ $\left(\mathbb{1} \otimes\left\langle\left. 0\right|_{k-l}\right) U_{k-l}^{\dagger} A_{l-1} U_{k-l}\left(\mathbb{1} \otimes|0\rangle_{k-l}\right)\right.$, so $Q_{l}$ here is simply the $l$ th spin, and $S_{l}$ contains only the gate $U_{l}$. The final operator $A_{L}$ so obtained acts only on the ancilla system, and the desired expectation is thus $\left\langle 0\left|A_{L}\right| 0\right\rangle$. At no stage in the procedure are we required to manipulate operators of dimension greater than $2 d \times 2 d$. To be entirely clear, a representative of each of these steps is depicted in Fig. 2 (a). A second example is provided by MERA circuits [10], which require that we manipulate operators of dimension at most $64 \times 64$. Here the sets $Q_{l}, S_{l}$ are defined with respect to levels of the MERA circuit and the

causal cone of the given Hamiltonian term. A first such step is represented in Fig. 2(b). After constructing $A_{l}$, we can move on to the next MERA layer (Fig. 2 (c)).

We now describe the new contraction procedure used to evaluate the more general expressions of Eq. (3), see Fig. 2. There are two sets of gates highlighted in these circuits, which will be dealt with in two separate contraction sequences, and we shall refer to these sets as the red cone (originating from the Hamiltonian as explained above) and blue cone (originating from the generator) respectively. The unhighlighted gates are simply cancelled as $U_{k}^{\dagger} U_{k}=\mathbb{1}$. We can hence restrict ourselves to the causal cones. We shall also refer to the gate $U_{j}$ whose generator we are calculating as the generatee.

The first contraction sequence involves the gates in the red cone. Here we set $A_{0}=H_{j, j+1}$ and proceed via a sequence of partial expectations, with the same sets $Q_{l}$, $S_{l}$ used in a standard contraction (see again Fig. 2 (b,c)). This continues until we reach the set $S_{G}$ containing the generatee $U_{j}$. At this point we are unable to continue as some or all of the gates in $S_{G}$ will have been cycled to the right-hand side (as we are calculating a partial trace). The final operator of this contraction $A_{G}$ is then constructed by conjugating the previous $A_{G-1}$ by those gates that have not been cycled. The second contraction focuses on the blue cone, and begins by initializing $B_{0}=|\mathbf{0}\rangle\langle\mathbf{0}|$, acting on the subsystem as the generatee. The contraction then iterates in the reverse order to the standard contraction,

$$
B_{j}=\operatorname{tr}\left[Q_{j}^{\prime}\left(\prod_{k \in S_{j}^{\prime}} U_{k}\right)\left(B_{j-1} \otimes|\mathbf{0}\rangle\langle\mathbf{0}|\right)\left(\prod_{k \in S_{j}^{\prime}} U_{k}\right)^{\dagger}\right],
$$

the primed $Q_{j}^{\prime}, S_{j}^{\prime}$ indicating the reversed order. This contraction also continues until it reaches the set $S_{G}$, at which point $B_{G}$ is constructed by (anti) conjugating $B_{G-1}$ with the gates from $S_{G}$ that have been cycled. The operator $F_{j}$ is then given by $F_{j}=\operatorname{tr}_{R_{j}}\left[B_{L} A_{L}\right]$, with $R_{j}$ as for Eq. (3). For clarity, a MERA procedure is shown in Fig. 2(d).

If the standard contraction procedure is efficient, then this modified procedure will also be efficient as the largest operators we must manipulate are defined by the same sets $S_{l}$. For example, determining the optimal generator for a gate in a staircase circuit acting on $N$ spins with ancilla dimension $d$ requires a time $O\left(N d^{3}\right)$, while for MERA the time required is $O(N \log N)$. The above methods can be readily applied to 2D settings of MERA [10], where one has, e.g., layers of Margolus partitionings as in a quantum cellular automaton [14], with a tree-like reduction of the number of sites in every second step; then again, the contraction of the two cones can be done efficiently.

Implementation. - We now have the main ingredients for an actual algorithm. Fig. 3 illustrates example implementations for the Heisenberg and critical Ising Hamiltonians

$$
H_{H}=-\frac{J}{2} \sum_{j=1}^{N} \sum_{k=1}^{3} \sigma_{j}^{k} \sigma_{j+1}^{k}, H_{I}=-\frac{1}{2} \sum_{j=1}^{N}\left(\sigma_{j}^{1} \sigma_{j+1}^{1}+\sigma_{j}^{3}\right)
$$




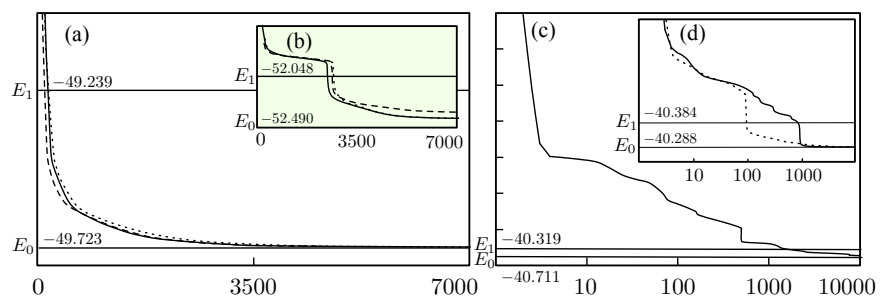

FIG. 3: (a) Staircase flow for the 30-qubit Heisenberg chain and (b) 28-qubit ring, in the number of flow steps. Plot (a) shows the decrease of expectation with the flow for $d=10$ (dashed), 20 (solid), 40 (dotted). Exact values for the two lowest eigenvalues (for $N=30$ ) are also shown. (c) MERA flow for the 32-qubit Ising chain. (d) MERA flow for the 32-qubit Ising ring and extended MERA.

as benchmarks. (a) and (b) illustrate an implementation for staircase circuits for the Heisenberg chain (with both open and closed boundary conditions) chosen for a first implementation as the corresponding ALPS-DMRG provides good benchmark. Each step of the flow requires the calculation and application of the optimal generator for each gate. We see that for open boundary conditions the staircase achieves, for the energy $E$, the same accuracy of $\Delta=\left(E-E_{0}\right) / E_{0}$ as the benchmark ALPS-DMRG to six significant digits, and no problems with local optima have been observed [16]. (c) presents a MERA implementation (representative when random initial conditions are drawn) for the 32-qubit critical Ising model for bond dimension 2. Even for this small bond dimension, the relative error of $\Delta=4.4696 \times 10^{-4}$ is achieved (note that this involves merely 61 unitaries acting on two spins, which is comparable in accuracy to DMRG for a dimension $d$ defining MPS being described by an order of magnitude of more real parameters), for the ring $\Delta=1.2901 \times 10^{-4}$. Similar performance is found for a 64 spin model. (d) For the extended MERA we find comparable performance but quicker convergence. We have also systematically compared the achievable accuracy for the Heisenberg model with MERA with bond dimension 2 (for which MERA performs slightly worse) with the one extended MERA where one appends an additional single layer of a quantum cellular automaton: This leads in instances to a significant improvement (of the order of $\left(E_{1}-E_{0}\right) / E_{0}$ in this model, critical in the thermodynamical limit), but, first and foremost, shows the flexibility of the approach [18].

Conclusions and further work. - We have shown how ideas from flow equations may be adapted to provide variational methods for approximating ground states of quantum many-body systems. The appeal of this approach is its flexibility as it is able to unify several existing ansatz classes within a single framework with a universal variational technique. Recent work into the dissemination of correlations in quantum many-body systems has stimulated much work on the construction of suitable variational classes. It is hoped that the methods presented here will facilitate the systematic exploration of the potential of these approaches.

Acknowledgments. - This work was supported by the DFG (SPP 1116), the EU (QAP), the EPSRC, QIP-IRC, Microsoft Research, EURYI, and the Nuffield foundation. We thank F. Verstraete and U. Schollwöck for discussions. Note added: For related work on MERA simulations that has appeared on the preprint server in the meantime, see Refs. [19].

[1] K.G. Wilson, Rev. Mod. Phys. 47, 773 (1975); S.R. White, Phys. Rev. Lett. 69, 2863 (1992).

[2] M. Fannes, B. Nachtergaele, and R.F. Werner, Lett. Math. Phys. 25, 249 (1992); S. Ostlund and S. Rommer, Phys. Rev. Lett. 75, 3537 (1995).

[3] G. Vidal, Phys. Rev. Lett. 91, 147902 (2003).

[4] F. Verstraete, D. Porras, and J.I. Cirac, cond-mat/0404706.

[5] F. Verstraete, J.J. Garcia-Ripoll, and J.I. Cirac, Phys. Rev. Lett. 93, 207204 (2004); F. Verstraete et al., cond-mat/0504305; T.J. Osborne, cond-mat/0605194, D. Perez-Garcia et al., Quant. Inf. Comp. 7, 401 (2007).

[6] U. Schollwöck, Rev. Mod. Phys. 77, 259 (2005).

[7] Matrix-product states approximate 1D ground states well if an (Renyi)-entropic "area law” [8] is satisfied.

[8] K. Audenaert et al., Phys. Rev. A 66, 042327 (2002); G. Vidal et al., Phys. Rev. Lett. 90, 227902 (2003); B.-Q. Jin and V.E. Korepin, J. Stat. Phys. 116, 79 (2004); M. Cramer and J. Eisert, New J. Phys. 8, 71 (2006); F. Verstraete and J.I. Cirac, Phys. Rev. B 73, 094423 (2006); M.B. Hastings, arXiv:0705.2024

[9] S. Anders et al., Phys. Rev. Lett. 97, 107206 (2006).

[10] G. Vidal, Phys. Rev. Lett. 99, 220405 (2007).

[11] C. Morningstar and M. Weinstein, Phys. Rev. D 54, 4131 (1996).

[12] F.J. Wegner, Ann. Phys. (Leipzig) 3, 77 (1994); S.D. Glazek and K.G. Wilson, Phys. Rev. D 48, 5863 (1993); S. Kehrein, Phys. Rev. Lett. 83, 4914 (1999).

[13] T. Schulte-Herbrüggen et al., Phys. Rev. A 72, 042331 (2005)

[14] B. Schumacher and R.F. Werner, quant-ph/0405174

[15] Eq. (3) provides us with the optimal generator at each flow point $t$, but not the optimal flow "strength" $2 / \lambda$. Poor choices of $\lambda$ may result in slow convergence. In the illustration presented below, $E(t)=\left\langle\mathbf{0}\left|U(t)^{\dagger} H U(t)\right| \mathbf{0}\right\rangle$ was well approximated, in the neighborhood of the optimum, by a quadratic in $1 / \lambda$ and heuristic techniques can make use of this to determine a good flow strength. The use of alternative techniques such as conjugate gradients or BFGS may be advantageous.

[16] Needless to say this method, as is in fact any other variational technique, is certifiable insofar one can find good lower bounds to the ground state energy. For a discussion of classical complexity, see Refs. [17].

[17] J. Eisert, Phys. Rev. Lett. 97, 260501 (2006); N. Schuch et al., ibid. 98, 140506 (2007); J. Kempe, A. Kitaev, and O. Regev, SIAM J. Comp. 35(5), 1070 (2006).

[18] We have also used a 1D-MERA with bond dimension 2 in 
a 2D Ising model, in an "snake-like" ordering of the spins. The achieved accuracy - although using essentially a 1D approach - is quite remarkable. In a $4 \times 4$ system the achieved accuracy is $\Delta=1.5513 \times 10^{-3}$, compared to DMRG energy of $\Delta=9.4412 \times 10^{-2}$. For larger systems, 1D-MERA outperforms 1D-DMRG even more distinctly.

[19] M. Rizzi and S. Montangero, arXiv:0706.0868; L. Cincio, J. Dziarmaga, and M.M. Rams, arXiv:0710.3829, G. Evenbly and G. Vidal, arXiv:0710.0692. 